\section{Computational Physics in Industry}

\section{B. Smit}

Shell Research and Technology Centre, Amsterdam

In an industrial context one rarely has the possibility to select the simplest systems for molecular simulations. Aims are set by business objectives rather than scientific convenience, and the imposed boundary conditions may require special attention. Increasing computer power may of course allow one in some cases to apply well-established and reliable fundamental methods to more complex systems. For some problems, however, the increase in computer time is prohibitively large so novel algorithms must be developed. It will be shown that a new Monte Carlo technique allows the simulation of systems of industrial interest that were considered impossible to study by molecular techniques. This development is illustrated by two examples: the adsorption of alkanes in zeolites and the phase behaviour of longchain alkanes.

\section{Adsorption in Zeolites}

Zeolites are crystalline inorganic polymers that have a well-defined microporous structure. These pores are accessible to various guest molecules. The large internal surface, the thermal stability and the presence of acid sites make zeolites an important class of catalytic materials for petrochemical applications.

Catalytic conversion inside the pores can be seen schematically as involving the absorption and diffusion of the reactants, catalytic conversion, and diffusion and desorption of products from the zeolite. The overall activity and selectivity of a particular reaction is the result of a delicate balance of these three processes. Much experimental and theoretical effort aims to understand each of the steps at a molecular level. The high selectivity of zeolites implies that the behaviour of the adsorbed molecules is system-specific. It is therefore essential to be able to study the behaviour of the adsorbed molecules of interest under reaction conditions. Unfortunately, this is very difficult, particularly for long-chain hydrocarbon molecules.

Computer simulations, used with molecular dynamics or Monte Carlo techniques, are an attractive alternative to experiments because they can, in principle, provide information for conditions under which experiments are not feasible.
However, simulations have been limited to atoms or small molecular guest molecules and could not be extended to molecules of catalytic relevance. For example, the diffusion of of the straight-chain alkanes butane and hexane from one channel to another in the zeolite silicalite is very slow and the rate of diffusion decreases with increasing chain length. Unacceptably long simulations are therefore required to obtain reliable results.

The Monte Carlo technique is not limited by the slow diffusion of molecules because diffusion barriers are by-passed by making moves to arbitrary positions in the zeolite. For chain molecules, however, this is not the case because the probability of finding a position without overlap between hydrocarbon and zeolite decreases exponentially with chain length. To be able to use Monte Carlo in this relatively complex system of industrial relevance it is necessary to develop novel algorithms which are orders of magnitude more efficient.

\section{Adsorption}

Consider adsorption of alkanes in zeolite. Fluids confined in narrow pores can have properties that are distinctly different from those of bulk fluids. Most studies have focused on simple fluids which can be modelled as near-spherical molecules. But molecular shape can also exert an influence on the fluid's behaviour, particularly for large, complex molecules.

Adsorption isotherms are of fundamental interest in studying fluids in pores because they may signal phase transitions, such as capillary condensation or wetting, of the fluid. For example, if a system exhibits capillary condensation, one would measure a stepped adsorption isotherm with hysteresis. As the pores of most zeolites are of molecular dimensions, adsorbed alkane molecules behave like a one-dimensional fluid. In a one-

dimensional system phase transitions do not generally occur so one would expect Type 1 adsorption isotherms for alkanes, that is, they do not show kinks or steps. If steps occur, they are usually attributed to capillary condensation in the exterior secondary pore system formed by the space between different crystals.

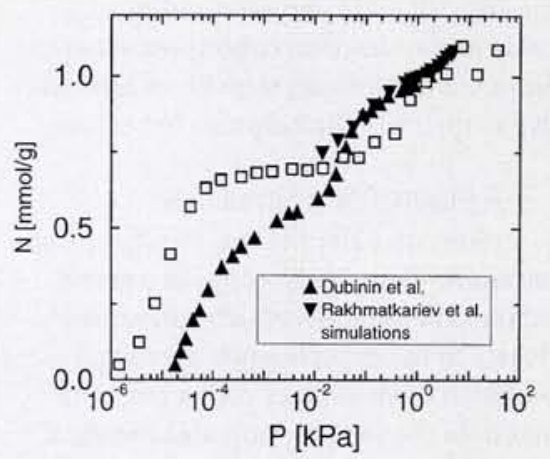

Fig. 1. Adsorption isotherms of heptane in silicalite. Closed symbols, experimental data; open symbols, results of simulations for a temperature $T=298 \mathrm{~K}$. The number of moles of gas $N$ absorbed per gram of silicalite is plotted as a function of the gas pressure $P$.
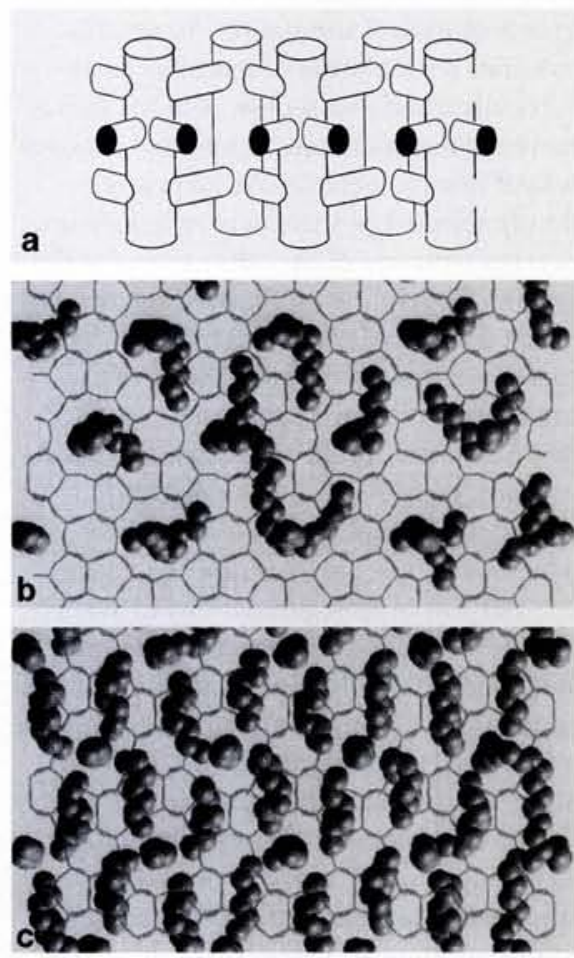

Fig. 2. Adsorption of alkanes in zeolite. A schematic illustration (a) of the pore structure of silicalite showing the wider straight channels and the narrow zig-zag channels. (BMC simulation of hexane in silicalite: b) approximately half the maximum loading; $\boldsymbol{c}$ ) almost maximum loading. The zig-zag channels are in the plane of the page; straight channels are perpendicular to the page. Grey spheres represent hexane molecules; $\mathrm{red} /$ orange lines represent the zeolite framework.

Adsorption isotherms have been determined for various $n$-alkanes in zeolite silicalite. They are indeed of Type 1 for the short-chain alkanes (methane and pentane). For hexane and heptane, however, a kink or step is observed suggesting that some kind of phase transition takes place, but no molecular explanation has been given. At present, data on sorption properties are scarce because experiments 
are difficult and time-consuming, especially under reaction conditions. Computer simulations seem to be an alternative to quantify the sorption behaviour.

\section{A Monte Carlo Simulation}

Molecular simulations of adsorption are conveniently performed in a grandcanonical ensemble, which corresponds closely to the experimental arrangement: a zeolite in open contact with a reservoir that fixes the temperature and chemical potential. The number of particles in the zeolite is allowed to fluctuate via Monte Carlo moves in which one attempts to add or remove a particle. Particularly for chain molecules, the insertion of molecules tends not to be favoured in a conventional grand-canonical simulation. To see this, consider a simulation of methane molecules adsorbed in a zeolite. Assume that a methane molecule can be inserted successfully if this molecule is placed at a randomly selected position where it does not overlap with one of the other atoms of the system. Depending on the loading, one needs on the order of 10 attempts to find such a position; for ethane this is of the order of 10, and for heptane as large as $10^{21}$. The latter would result in a simulation of many years even for a super-computer, and allowing simulations to equilibrate would be prohibitively time-consuming.

For this reason simulations of adsorption have focused on systems containing small molecules. To make grand-canonical simulations of long-chain alkanes possible, one can use the configurational-bias Monte Carlo (CBMC) technique (see insert) for the insertion step. Simulated adsorption isotherms of various alkanes in silicalite obtained using CBMC and a unified atom model (see insert) have been compared with experimental data [4]. For butane a Type I isotherm is observed, and the agreement between experiments and simulation is good. For hexane and heptane, there is reasonable agreement at high pressures, but at low pressures deviations exist which indicate that the zeolite-alkane model can be further improved.

More importantly, for heptane both the experiments and the simulations show a step at approximately half the loading (Fig. 1). Detailed inspection of the calculated adsorption isotherm shows a kink at this loading for hexane. As the simulations are performed on a perfect single crystal, these deviations from the Type I isotherm must be due to a transition of the fluid inside the pores and cannot be attributed to the secondary pore system.

\section{Configurational-bias Monte Carlo}

In configurational-bias Monte Carlo [1] a molecule is not inserted at random but is inserted atom-by-atom in such a way that overlaps are avoided. This "growing" procedure introduces a bias which leads to an incorrect distribution of configurations. This bias can be removed exactly [2] by using the weight introduced by Rosenbluth and Rosenbluth [3] to correct the biasing of acceptance of trail conformations and not the thermal averages as was originally proposed. The probability of generating a particular confirmation is then proportional to the conformation's Boltzmann weight, thus removing the main drawback of the original Rosenbluth scheme.

A simulation is performed in cycles, and each cycle consists of a number of randomly selected moves: displacement of particles, rotation of particles, partial regrowing of a molecule, and regrowing of a molecule at a randomly selected position. The figure illustrates the growing of an alkane in a zeolite in a CBMC move. The black circles represent the atoms of the zeolite, and the white circles the atoms of the alkane. Seven atoms have

\section{United-Atom Model}

In a united-atom model for alkane molecules the $\mathrm{CH}_{3}$ and $\mathrm{CH}_{2}$ groups are considered as single interaction centres, with pseudoatoms in a given chain connected by rigid bonds. Non-bonded interactions are described by truncated Lennard-Jones potentials; intramolecular interactions include bond-bending and torsion. The zeolite is modelled as a rigid crystal and alkane - zeolite interactions are

Silicalite has two types of channels, straight and zig-zag, which are connected via intersections (Fig. 2a). Figs. $2 \mathrm{~b}$ and c shows "snapshots" of hexane molecules in the channels of silicalite at two different loadings: Fig. $2 \mathrm{~b}$ is at approximately half the maximum loading and in Fig. $2 \mathrm{c}$ the zeolite is almost saturated. Note that the length of a hexane molecule is of the order of the length of the period of the zig-zag channel. At low chemical potential, the hexane molecules move "freely" in these channels and the molecules will be in the intersections for some of the time. If part of the intersection is occupied, other molecules cannot reside in the straight channels at the same time.

At high pressures, almost all the hexane molecules fit exactly into the zig-zag channel (Fig. 2C): they no longer move freely and keep their noses and tails out of the intersection. In such a configuration the entire straight channel can be tightly packed with hexane molecules. This explains the plateau in the adsorption isotherm: in order to fill the entire zeolite

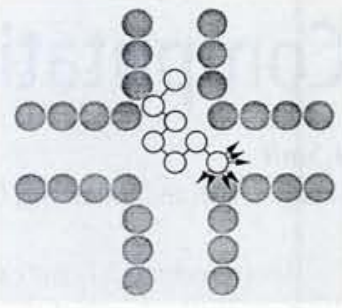

been grown successfully, and an attempt is made to insert the eighth. The arrows indicate seven trial positions for which the energy $u_{i}$ is calculated. One is selected with a probability given by $p_{j}=\exp \left(-u_{i} / k_{\mathrm{B}} T\right) / w_{\text {new }}(l)$ where $w_{\text {new }}(l)=\Sigma_{j} \exp \left(-u_{j} / k_{\mathrm{B}} T\right)$ for a temperature $T$ and Boltzmann constant $k_{\mathrm{B}}$. Similarly, for the old configuration, one calculates $w_{\text {old }}(l)=$ $\sum_{i} \exp \left(-u_{i} / k_{\mathrm{B}} T\right)$. This is repeated until the entire chain of length $m$ has been grown. The bias of the growing is removed by the replacement of $\exp \left(-\Delta U / k_{\mathrm{B}} T\right)$ by $\prod_{l=1}^{m} w_{\text {new }}(l) / \prod_{l=1}^{m} w_{\text {old }}(l)$ in the acceptance rule. This Monte Carlo technique can be 10 to 50 orders of magnitude more efficient than molecular dynamics (the number of trial orientations in the CMBC moves for the adsorption simulations ranged from five for butane to eight for heptane).

assumed to be dominated by dispersive interaction with oxygen atoms which are described by a Lennard-Jones potential. Size and energy parameters are fitted to the heats of adsorption and the Henry coefficients of $n$-alkanes in silicalite. Simulations of the absorption isotherms used periodic boundary conditions for a box contained 16 unit cells of silicalite with total size $40.14 \times 39.840 \times 53.680 \AA^{3}$.

structure neatly, the hexane molecules located in zig-zag channels have first to be frozen in these channels. This freezing of the positions of the hexane molecules in the channels implies a loss of entropy, so an increased pressure or chemical potential is needed. This increase is reflected as a step in the adsorption isotherm.

The incommensurate freezing of $n$ alkanes which just fit into the zig-zag channels has been confirmed recently using temperature programmed desorption [5]. In contrast to other alkanes, both heptane and hexane show a second desorption peak at low temperatures corresponding to desorption of the low entropy molecules found in the zig-zag channels at high loadings.

Traditionally, research on the applications of zeolites in the petrochemical industry has focused on trying to understand catalytic conversion in the pores of the zeolite; less attention has been paid to the sorption properties per se. This lack of knowledge has long hampered the understanding of phenomena such as the 
"compensation effect" - an unexpectedly high conversion of long-chain alkanes seen in catalytic cracking which has only recently been attributed to differences in the sorption behaviour of the alkanes. The example described above shows that quantifying sorption using computational techniques such as configurational-bias Monte Carlo is not only a prerequisite for the design and optimization of catalysts, but also leads to a better understanding of the fundamentals of zeolite catalysis.

\section{Phase Equilibria}

Alkanes are thermally unstable above approximately $650 \mathrm{~K}$, which makes experimental determination of the critical point of alkanes longer than decane extremely difficult. The longer alkanes, however, are present in mixtures of practical importance for the petrochemical industry. The number of components can be so large that it is impractical to determine all phase diagrams experimentally. One has therefore to rely on predictions made by equations of state where the parameters are directly related to the critical properties of the pure components. Data are scarce and contradictory, so semi-empirical methods are used to estimate the critical properties.

Configurational-bias Monte Carlo has been combined with the Gibbs-ensemble technique (see insert) to simulate the vapour-liquid equilibria of the $n$-alkanes at conditions where experiments are not (yet) feasible.

Most models for the vapour-liquid equilibrium of alkanes have been obtained by fitting simulation data to experimental properties of the liquid at standard conditions. Since the phase diagrams are very sensitive to the choice of interaction potentials, models can be improved by fitting predicted critical properties to experi-

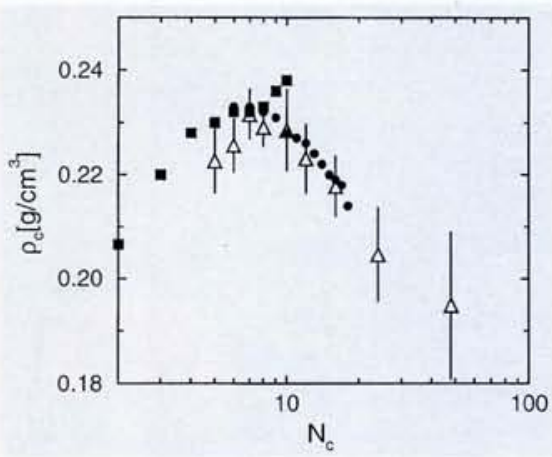

Fig. 3. The critical density of $n$-alkanes as a function of the carbon number $N_{c}$. The open symbols for the simulation data show a maximum at $N_{\mathrm{c}} \approx 8$ and the closed symbols are for two sets of experimental data, one of which shows a plateau at $N_{\mathrm{c}}=8$.

\section{CBMC and the Gibbs Ensemble}

The Gibbs-ensemble scheme was introduced [6] as an efficient tool to simulate vapour-liquid phase equilibria, where the simulations of the phases are carried out in parallel. Monte Carlo rules which allow for changes in the number of particles and the volume ensure that the two boxes representing the two phases are in thermodynamic equilibrium with each other. Since the two boxes are not in "physical contact", there is no interface and the bulk properties of the two coexisting phases can be obtained directly with a surprisingly small number of particles.

This makes the Gibbs ensemble extremely

\section{Beyond Chain Molecules}

CBMC is not simply a method for generating conformations of chain molecules since it is much more general. It can be used as a scheme to perform collective rearrangements of any set of labelled coordinates. In fact, the scheme can be used to carry out Monte Carlo moves to swap $n$ small particles within a volume $\Delta V$ with one large particle that occupies the same (excluded) volume. This application has been exploited to study mixtures of large and small hard spheres [T. Biben, unpub.]. Gibbs-ensemble simulations of mixtures of spherical colloids and rod-like polymers have been performed [9] using CBMC-style particle swaps and a closely related approach was used to study phase separation in mixtures of hardcore particles on a lattice [10]. Applying CBMC to improve the sampling of ionic solutions has been proposed [11].

A different application of the CBMC ideas involves an algorithm to perform Monte Carlo moves in parallel has been developed by Esselink et al. [12]. Parallel Monte Carlo appears to be a contradiction in terms, since the Monte Carlo procedure is an intrinsically sequential

mental data. This results in simulations which reproduce critical temperatures fairly well [7]. However, this is not the case for the critical density, largely because there is considerable disagreement between the experimental data.

Much of the current knowledge on the critical properties of the higher alkanes is based on extrapolating experimental data, usually as a montonically increasing function of the carbon number. CBMC simulations predict a maximum critical density at about $\mathrm{C}_{8}$ (Fig. 3). Some experimental data give a plateau at $\mathrm{C}_{8}$ while other show no evidence of a maximum. It is interesting to note that Monte Carlo simulations of the vapour-liquid curve of a polymeric bead-spring model for various chain lengths [8] show a decrease of the critical density as a function of chain length. This phenomenon is a general feature of longchain molecules, as has already been pointed out by Flory. efflcient for phase equilibrium calculations. The major limitation is that one of the steps involves the exchange of particles between the two boxes. For liquids consisting of small molecules this does not cause serious problems. However, for chain molecules the probability of successful exchanges can become very small. For example, under conditions where it takes approximately $10^{3}$ attempts per successful exchange of methane, it takes of the order of $10^{3 n}$ attempts for an $n$-alkane. However, the method can be made to work for much longer chain molecules by combining the Gibbs-ensemble method with CBMC.

process: one has to know whether the current move is accepted or rejected before continuing with the next move. The conventional way of introducing parallelism is to distribute the energy calculation over various processors, or to farm out the calculation by performing separate simulations over the processors.

Although the latter is extremely efficient and requires minimum skills, it is not a truly parallel algorithm. For example, farming out a calculation is not very efficient if the equilibration of the system takes a significant amount of processor time. In the algorithm of Esselink et al. several trial positions are generated in parallel and the one with the highest probability of being accepted is selected with the highest probability. This selection step introduces a bias which is removed by adjusting the acceptance rules. The generation of each trial move, which includes the calculation of the energy (the Rosenbluth factor in the case of chain molecules), is distributed over the various processors. The approach has been used to calculate phase equilibrium in parallel using the Gibbs-ensemble technique [13].

\section{References}

[1] B. Smit \& J.I. Siepmann Science 264 (1994) 1118; D. Frenkel \& B. Smit, Understanding Molecular Simulations (Academic Press, 1996).

[2] D. Frenkel, G.C.A.M. Mooij \& B. Smit, J. Phys.:

Cond. Matter 4 (1992) 3053.

[3] M.N. Rosenbluth \& A.W. Rosenbluth, J. Chem.

Phys. 23 (1955) 356.

[4] B. Smit \& T.L.M. Maesen, Nature 374 (1995) 42.

[5] W.J.M. van Well et al., Angew. Chem. (Int. Ed.) 34 (1995) 2543.

[6] A.Z. Panagiotopoulos, Mol. Phys. 61 (1987) 813. [7] J.I. Siepmann, S. Karaborni \& B. Smit, Nature 365 (1993) 330; B. Smit, S. Karaborni \& J.I. Siepmann, J. Chem. Phys, 102 (1995) 2126.

[8] G.C.A.M. Mooij, D. Frenkel \& B. Smit, J. Phys.: Cond. Matter 4 (1992) L255; Y.-J. Sheng et al., Macromolecules 27 (1994) 400.

[9] P. Bolhuis \& D. Frenkel, J. Chem. Phys. 101 (1995) 9869.

[10] M. Dijkstra \& D. Frenkel, Phys. Rev. Lett. 72 (1994) 298; M. Dijkstra, D. Frenkel \& J.-P. Hansen, J.

Chem. Phys. 101 (1994) 3179.

[11] J.C. Shelley \& G.N. Patey, J. Chem. Phys. 100 (1994) 8265 .

[12] K. Esselink, L.D.J.C. Loyens \& B. Smit, Phys. Rev. E 51 (1995) 1560.

[13] L.D.J.C. Loyens, B. Smit \& K. Esselink, Mol. Phys. 86 (1995) 171. 